\title{
CAPÍTULO 46: USO DE ÓLEO ESSENCIAL DE PIMENTA ROSA (Schinus terebinthifolius Raddi) INCORPORADO EM REVESTIMENTOS PARA CONTROLE DE MICRORGANISMOS - REVISÃO
}

\section{CHAPTER 46: USE OF ESSENTIAL OIL OF PINK PEPPER INCORPORATED IN COATING FOR MICROORGANISM CONTROL - REVIEW}

Daniele Pereira do Amaral ${ }^{1}$; Isamara Reis Gomes ${ }^{2}$; Daniel Saraiva Lopes ${ }^{3}$ Lethycia Dos Santos Vieira da Matta $^{4}$.

\begin{abstract}
Resumo
Revestimentos e filmes ativos são técnicas inovadoras de conservação de alimentos e estas têm buscado constantemente por agentes ativos naturais compatíveis com produtos mais saudáveis. O uso dos óleos essenciais como agentes antimicrobianos naturais substitutos de aditivos artificiais, tem sido uma alternativa eficiente no controle de microrganismos. O óleo essencial de pimenta rosa tem sido identificado como um eficiente agente antimicrobiano, com ação comprovada no controle de crescimento de bactérias in vitro e após a sua incorporação em filmes de embalagens. A sua aplicação ainda não foi testada em trabalhos de desenvolvimento de revestimentos ativos aplicados na conservação de alimentos, sendo necessária a realização de investigação de concentrações efetivas para controle de bactérias e fungos, notadamente nos produtos de origem vegetal que são armazenados in natura e sofrem grandes perdas pós-colheita pelo ataque de fungos.
\end{abstract}

Palavras-Chave Schinus terebinthifolius Raddi, revestimento ativo, óleo essencial, pimenta rosa.

\begin{abstract}
Active coatings and films are innovative food preservation techniques and they are constantly looking for natural active agents compatible with healthier products. The use of essential oils as natural antimicrobial agents replacing artificial additives, has been an efficient alternative in the control of microorganisms. The essential oil of pink pepper has been identified as an efficient antimicrobial agent, with proven action in controlling the growth of bacteria in vitro and after its incorporation in packaging films. Its application has not yet been tested in the development of active coatings applied to food preservation, requiring the investigation of effective concentrations to control bacteria and fungi, notably in products of plant origin that are stored in natura and suffer great post-harvest losses due to fungi attack.
\end{abstract}

Keywords: Schinus terebinthifolius Raddi, active coating, essential oil, pink pepper.

\footnotetext{
${ }^{1}$ Doutora, Universidade Estadual do Norte Fluminense - UENF, dani.alimento@ gmail.com.br

${ }^{2}$ Mestranda em Produção Vegetal, Universidade Estadual do Norte Fluminense -UENF, isamarareisgomes@gmail.com.br

${ }^{3}$ Tecnólogo em Alimentos, Instituto Federal Fluminense, danielsaraiva15.ds@ gmail.com.br

${ }^{4}$ Biologia, Universidade Estadual do Norte Fluminense - UENF, lethyciadossantostab@gmail.com.br
} 


\section{Introdução}

A busca por novas técnicas que minimizem as perdas e auxiliem no controle de microrganismos que causam a deterioração tem sido o foco de muitas pesquisas na área de armazenamento de alimentos. Este fato está diretamente relacionado a um mercado consumidor mais competitivo e que busca alimentos saudáveis e com características mais próximas do alimento in natura (CALO et al., 2015).

As embalagens tradicionais apresentam apenas a função passiva de proteção do alimento, no entanto, técnicas inovadoras vêm sendo estudadas a fim de promover uma interação benéfica das substâncias que compõem a embalagem diretamente com o alimento, tais como, os filmes e revestimentos ativos (HAFSA et al., 2016; HAN, YU and WANG, 2014). Estas técnicas possuem como principais objetivos o aumento da validade comercial e a diminuição do índice de contaminação dos alimentos, principalmente as frutas e vegetais, que apresentam um curto período de comercialização, com elevadas perdas pós-colheita que impactam sobre a elevação do seu custo (AMARAL, 2014).

De acordo com FDA (2015), os revestimentos comestíveis devem ser seguros para uso em alimentos, e de forma geral, os revestimentos têm como base, materiais biológicos, tais como, proteínas, lipídios e polissacarídeos, sendo os mais comuns o amido, a celulose e a pectina, que são de origem natural, comumente conhecidos como biopolímeros (TZOUMAKI, BILIADERIS, VASILAKAKIS, 2009). O uso de biopolímeros naturais aplicados sobre alimentos tem apresentado grande interesse, não somente por parte de pesquisadores, mas também de produtores e comerciantes de frutas e hortaliças, isso porque o uso desses materiais de origem natural ajuda a minimizar o impacto ambiental e reduzir as perdas pós-colheita (GARCIA et al., 2010; ANSORENA; MARCOVICH; ROURA, 2011; VU et al., 2011; WRÓBLEWSKA-KREPSZTUL, et al., 2018).

A incorporação de compostos ativos naturais nos revestimentos e filmes que promovem alterações benéficas ao alimento tem sido uma alternativa ao uso de substâncias químicas, estimulando uma busca constante por produtos naturais que atuem de forma positiva para a saúde do consumidor (HAFSA et al., 2016).

Existem diversos compostos naturais que são utilizados como agentes antimicrobianos como os óleos essenciais (OE), os agentes antioxidantes como o ácido ascórbico, dentre outros, esses compostos trazem ao revestimento, além da função de barreira, a função de interação e de proteção do alimento contra agentes de deterioração, melhorando as propriedades funcionais (ZAHID et al., 2012). 
Os OE são extratos de origem vegetal que possuem em sua composição, substâncias bioativas (ASBAHANI et al., 2015). A planta Schinus terebinthifolius Raddi, conhecida como Aroeira, é uma planta nativa do Brasil, amplamente distribuída em toda América do Sul (AGRA et al., 2008). Seu fruto, popularmente conhecido como Pimenta Rosa, tem sido muito utilizado na indústria de alimentos devido ao seu sabor e aroma característico (ULIANA et al., 2016). O seu extrato tem sido considerado de alta eficiência como agente antimicrobiano e antioxidante. Entretanto, poucos trabalhos têm abordado o seu uso e a aplicação de seu OE em revestimentos de alimentos (DANNENBERG, et al. 2016).

O objetivo deste trabalho foi identificar o estado da arte na utilização do óleo essencial de pimenta rosa em revestimentos ativos e sua ação como agente antimicrobiano, tendo em vista a sua aplicação no desenvolvimento de revestimentos ativos para controle de microrganismos durante o armazenamento de alimentos.

\section{Importância das embalagens}

O conceito tradicional das embalagens para alimentos tem por premissa a função de preservar as características físicas, sensoriais, nutricionais e sanitárias dos alimentos, durante o período de comercialização (AMARAL, 2014). O conceito mais moderno vai além da função básica da embalagem, que além da função passiva de preservação e contenção, ela deve incluir aspectos de segurança, conveniência e perigo ao meio ambiente (DAINELLI et al., 2008; HAN, YU and WANG, 2014).

As embalagens ativas e Inteligentes promovem uma maior interação com o alimento e uma melhoria na preservação da qualidade do produto, bem como o aumento de sua validade comercial e segurança do alimento (GANIARI, et. al., 2017; REALINI, MARCOS, 2014; BIJI et al., 2015). Segundo o Guia da União Europeia para o Regulamento da Comissão $\mathrm{n}^{\circ} 450 / 2009$, a embalagem é denominada ativa quando fornece funções além daquelas tradicionais de proteção e de barreira inerte ao ambiente externo (UE, 2009).

As embalagens ativas podem funcionar como filmes ou como revestimentos. Os filmes consistem em películas pré-formadas e secas, compostas por uma matriz polimérica que envolve os alimentos, podendo ser incorporada ou não com agentes ativos. Já os revestimentos consistem em um gel formado por uma matriz polimérica, incorporado ou não com agentes ativos, que é aplicado na superfície do alimento (FALGUERA et al, 2011). 


\section{Importância dos revestimentos ativos}

As coberturas comestíveis ou revestimentos são definidos como uma fina camada de material comestível, depositada sobre a superfície do alimento, que tem a finalidade de inibir e/ou reduzir a perda de umidade, promover barreiras semipermeáveis à migração de solutos, às trocas gasosas, à respiração, bem como a supressão de distúrbios fisiológicos (WONG et al., 1994, BALDWIN et al., 1996; ROJAS-GRAU, TAPIA, MARTÍN-BELLOSO, 2008). Os revestimentos podem atuar também como carreadores de agentes antimicrobianos e agentes de controle de firmeza, ou até mesmo de compostos químicos que melhorem a estabilidade química e inibam as reações oxidativas (DAMODARAN; PARKIN; FENNEMA, 2010).

Diversos compostos podem ser usados na formulação dos revestimentos ativos, como polissacarídeos, proteínas, lipídios ou a combinação destes compostos. O uso de materiais provenientes de fontes renováveis, capazes de se decomporem naturalmente no ambiente, com menos efeitos ambientais, tem sido a motivação dessa proposta de inovação tecnológica, embora seu uso ainda apresente algumas limitações devido ao seu baixo desempenho, principalmente no que se refere às propriedades de barreira (SUKHIJA, SINGH, RIAR, 2016; MAJID et. al., 2018). O uso destes materiais se deve à demanda crescente por parte dos consumidores por alimentos seguros e saudáveis, que em muitos casos podem ser uma alternativa às embalagens sintéticas (ARNON-RIPS and POVERENOV, 2018).

Os trabalhos de desenvolvimento de biopolímeros têm sido amplamente difundidos e estão abrindo perspectivas para as inovações tecnológicas em revestimentos (ESPITIA et al., 2014). Os amidos em geral, têm o principal polímero usado nas formulações de revestimentos comestíveis (HENRIQUE; CEREDA; SARMENTO, 2008), tendo como principais características a boa transparência, o incremento de brilho superficial, boa resistência às trocas gasosas e também por permitir a adição de compostos ativos em sua formulação, podendo assim melhorar suas propriedades funcionais, com isso apresentando elevado potencial de uso na indústria de alimentos como alternativa na conservação (PELISSARI, et al., 2019; AHMED et al., 2017).

As propriedades especiais dos revestimentos ativos são obtidas através da incorporação direta de compostos ativos (aditivos) à matriz polimérica, conferindo propriedades específicas ao revestimento, apresentando grande potencial de uso, seja por liberar/emitir os agentes ativos (antioxidantes, antimicrobianos), reter compostos (etileno, oxigênio, água) ou neutralizar compostos indesejáveis produzidos no alimento (RESTUCCIA et al., 2010; ZANETTI et al., 2015; YILDIRIM et al., 2018). 


\section{Perspectivas do uso de óleos essenciais}

Os OE são extratos de origem vegetal que possuem em sua composição, substâncias bioativas sintetizadas pelas plantas (ASBAHANI et al., 2015). Esses extratos vegetais têm despertado grande interesse na indústria de alimentos em função da eficácia de seus componentes químicos naturais que apresentam atividades antimicrobianas e antioxidantes, agregando também valor aos produtos alimentícios em função do apelo de consumo de um produto conservado com substâncias naturais, podendo com isso serem utilizados como alternativa aos conservantes sintéticos (CALO et al., 2015; DANNENBERG et al., 2016).

Boa parte dos OE extraídos de plantas, condimentos e especiarias apresentam propriedades antimicrobianas e antioxidantes, o que possibilita seu uso na indústria de alimentos (VIUDA-MARTOS et al., 2010; ATARÉS and CHIRALT, 2016; VILELA et al., 2018). Por serem fitoquímicos naturais os OE são antimicrobianos naturais encontrados em muitas plantas, sendo eficaz em diversas aplicações, tais como, para eliminar diminuindo e/ou impedir o crescimento e a sobrevivência de microrganismos patogênicos (JUNEJA, DWIVEDI, YAN, 2012; CALLAWAY et al., 2011; LI et al., 2011, MUTHAIYAN, LIMAYEM, RICKE, 2011; ROLLER and LUSENGO, 1997, TIWARI et al., 2009).

Essas substâncias naturais (OE), derivam diretamente de um sistema biológico sem sofrer alterações ou modificações em um ambiente de laboratório são conhecidas como antimicrobianos naturais (LI et al., 2011, LÓPEZ-MALO, ALZAMORA, GUERRERO, 2000, SIRSAT, MUTHAIYAN, RICKES, 2009; NANNAPANENI et al., 2009).

Nos últimos anos, diversos artigos de revisão foram publicados sobre a incorporação de compostos antimicrobianos como os OE em materiais de embalagem, tendo destaque a eficácia da substância liberada na redução da deterioração de alimentos (SUNG et al., 2013). Acredita-se que sua ação esteja associada as nanomoléculas antimicrobianas que compõem os $\mathrm{OE}$, que supostamente inibem a respiração microbiana e aumentam a permeabilidade da membrana plasmática o que pode induzir a morte celular (AUMEERUDDY-ELALFI, GURIB-FAKIM and MAHOMOODALLY, 2015).

No entanto, seu uso diretamente sobre o alimento é muitas vezes limitado devido ao odor e sabor forte, que podem vir a causar modificações nas características sensoriais dos alimentos, podendo limitar a aceitação do produto pelos consumidores (RUIZNAVAJAS et al., 2013; ATARÉS and CHIRALT, 2016). 


\section{Potencial de uso do óleo essencial de pimenta rosa}

A planta Schinus terebinthifolius Raddi, conhecida no Brasil como Aroeira, pertence à família Anacardiácea e é uma planta nativa do Brasil, sendo amplamente distribuída em toda América do Sul, principalmente no litoral brasileiro (AGRA et al., 2008). Seu fruto comumente conhecido como Pimenta Rosa, tem sido nos últimos anos muito utilizados na indústria de alimentos devido ao seu sabor e aroma característico (ULIANA et al., 2016), além disso, o estudo da sua eficiência como agente antimicrobiano e antioxidante foi relatado na literatura por Uliana et al., 2016 (in vitro), Dannenberg, Funck, Mattei, Silva e Fiorentini, 2016 (in situ), Gois et al., 2016 (in vivo), e em embalagens ativas utilizando óleo essencial de pimenta rosa (OEPR) como componente antimicrobiano (DANNENBERG et al., 2017).

Os OE são misturas complexas de compostos voláteis que apresentam atividade antimicrobiana, atuando na planta como um mecanismo de defesa natural frente a condições adversas (ASBAHANI et al., 2015; DANNENBERG et al., 2017). Estudos realizados por Cavalcanti et al. (2015) e Gois et al. (2016), identificaram que a composição química do óleo essencial de pimenta rosa (OEPR) é composto predominantemente por mono terpenos, tais como: $\alpha$-pineno, $\beta$-pineno, mirceno e limoneno. Na maioria dos casos, o processo de extração dos OE é realizado com solventes orgânicos, ou arraste a vapor, e por serem de origem de plantas comestíveis são considerados como GRAS (Generally Recognized As Safe) pelo FDA (Food and Drug Administration) dos Estados Unidos, podendo com isso serem usados como aditivos alimentares (BURT, 2004; ASBAHANI et al., 2015; CALO et al., 2015; GHABRAIE et al., 2016).

A ação antimicrobiana dos OE pode estar associada aos seus constituintes ativos (HYLDGAARD, MYGIND, MEYER., 2012), onde pequenas moléculas de substâncias antimicrobianas entram na célula, interagindo principalmente com o mecanismo de síntese de proteínas (KHANEGHAH, et al., 2018), atuam diretamente sobre camada externa da bactéria, causando sua desnaturação, e consequentemente sua morte (NAZZARO, FRATIANNI, and MARTINO, 2013; RAI et al., 2017). A atividade antimicrobiana do óleo essencial de pimenta rosa é atribuída aos seus componentes majoritários como $\beta$-mirceno (41\%), $\beta$-cubebeno (12\%) e limoneno (9\%), que são antibacterianos conhecidos, ou um possível efeito sinérgico entre esses compostos e os compostos minoritários que compõem o óleo essencial de pimenta rosa (NGUEFACK et al., 2012; DANNENBERG et al., 2019). Resultado próximo ao obtido por Dannenberg et al. (2019) foi obtido por Gois et al. (2016), onde encontrou as concentrações de 12,36\% 
de limoneno e 10,36\% de $\alpha$-pineno em OE de frutos de S. terebinthifolius Radd. Já Uliana et al. (2016), relatou a ocorrência de $6,78 \%$ de mirceno e 4,05\% de $\alpha$-pineno em OE extraído de folhas dessa espécie (S. terebinthifolius Radd). Cavalcanti et al. (2015) ao estudar o OE obtido dos frutos de S. terebinthifolius, obteve como compostos predominantes $o$-pineno (44,9\%), $\beta$-pineno $(15,1 \%)$ e germacreno D $(17,6 \%)$.

\section{Revestimentos contendo óleo de Pimenta Rosa}

Diversos estudos vêm sendo realizados a fim de avaliar o efeito dos OE como conservantes naturais, seja por meio de sua incorporação no filme da embalagem (matriz polimérica) ou diretamente na incorporação ao produto alimentício, obtendo-se efeitos favoráveis na conservação dos alimentos (DANNENBERG et al., 2016; DANNENBERG et al., 2017). No entanto, a sua aplicação direta sobre o alimento pode afetar as características sensoriais, o que não é desejado, principalmente no caso de revestimentos de frutos e hortaliças que podem sofrer alterações de suas características organolépticas de sabor e odor (ATARÉS and CHIRALT, 2016; GHABRAIE et al., 2016).

Uma forma de se minimizar o efeito de alteração de sabor e odor nos produtos consiste em realizar a incorporação do óleo essencial de pimenta rosa nos filmes e revestimentos, que além de reduzir tal efeito negativo, também proporciona uma liberação dos compostos ativos gradualmente durante o período de armazenamento, além do que, esses compostos se concentram na parte externa do alimento, onde o grau de contaminação é maior, apresentando assim maior eficiência no controle microbiano (ASBAHANI et al., 2015; DANNENBERG et al., 2017).

O óleo de pimenta rosa tem demonstrado efeito de inibição de crescimento in vitro de bactérias dos grupos L. monocytogenes e E. coli, dois tipos de bactérias de grande importância de contaminação de produtos na indústria de alimentos (DOURADO et al., 2012). Este efeito de inibição de bactérias foi verificado também no trabalho de DANNENBERG et al. (2017), em que constatou redução significativa no crescimento de S. aureus, L. monocytogenes, E. coli e S. Typhimurium, ao avaliar a ação do OE de pimenta rosa incorporado a filmes ativos de acetato celulose nas concentrações $0,2,4$ e $6 \%(\mathrm{v} / \mathrm{v})$.

Ao contrário, no estudo realizado por Uliana et al. (2016), não se observou o efeito de inibição do crescimento de E. coli e S. aureus, mesmo nas maiores concentrações testadas, de $1 \mathrm{mg} / \mathrm{mL}(0,1 \%)$, para o EO das folhas de S. terebinthifolius. Resultado semelhante foi obtido por Aumeeruddy-elalfi, Gurib-Fakim and Mahomoodally (2015), não onde não observou atividade antimicrobiana para a OE de S. terebinthifolius contra 
cepas de $E$. coli e $P$. aeruginosa, mas indicaram que em $1 \mathrm{mg} / \mathrm{mL}(0,1 \%)$ se obtinha a concentração mínima bacteriana para $S$. aureus.

Segundo Dannenberg et al. (2019), o óleo de pimenta rosa é efetivo na inibição completa dos microrganismos S. aureus e L. monocytogenes quando aplicada na concentração de 2,72 $\mathrm{mg} \mathrm{mL}^{-1}$ em diferentes meios de cultura, destacando o seu grande potencial de utilização como agente antimicrobiano natural. Em estudos realizados por Braga et al. (2007) e El-Massry et al. (2009) ao avaliar a eficiência antimicrobiana de extrato e do óleo essencial de pimenta rosa sobre bactérias gram-positivas, constataram eficiência destes compostos, frente as bactérias, onde sua concentração mínima inibitória foi menor que $>1000 \mu \mathrm{g} / \mathrm{mL}$.

\section{Considerações Finais}

Este trabalho constatou a grande evolução da utilização de OE como agente ativo aplicado no desenvolvimento de biofilmes e revestimentos aplicados na conservação de alimentos, agregando os efeitos antimicrobianos e antioxidantes, porém existe restrição ao seu uso devido ao eventual efeito de interferência nas características organolépticas de sabor e odor dos alimentos.

No caso específico do óleo de pimenta rosa verificou-se também que ele tem grande efeito de ação antioxidante e antimicrobiano, com ação comprovada no controle de crescimento de bactérias após a sua incorporação em filmes de embalagens. A sua aplicação ainda não foi testada em trabalhos de desenvolvimento de revestimentos ativos aplicados na conservação de alimentos, sendo necessária a realização de investigação de concentrações efetivas para controle de bactérias e fungos, notadamente nos produtos de origem vegetal que são conservados in natura e sofrem grandes perdas pós-colheita pelo ataque de fungos.

\section{Referências}

AGRA, M. F.; SILVA, K. N.; BASÍLIO, I. J. L. D.; FREITAS, P. F.; BARBOSA-FILHO, J. M. Survey of medicinal plants used in the region northeast of Brazil Review. Brazilian Journal of Pharmacognosy, v.18, p. 472-508, 2008.

AHMED, I.; LIN, H.; ZOU, L.; BRODY, A. L.; LI, Z.; QAZI, I. M.; PAVASE, T. R.; LV, L. A comprehensive review on the application of active packaging technologies to muscle foods - Review. Food Control, v. 82, p. 163-178, 2017.

AMARAL, D.P. Revestimento Ativo antiescurecimento a base de proteína de soro de leite. Orientadora: Nathália Ramos de Melo. 2014. 59 f. Dissertação (Mestrado em Ciência e Tecnologia de Alimentos). Universidade Federal Rural do Rio de Janeiro, UFRRJ, 2014. 
ANSORENA, M. R., MARCOVICH, N. E. and ROURA, S. I. Impact of edible coatings and mild heat shocks on quality of minimally processed broccoli (Brassica oleracea L.) during refrigerated storage. Postharvest Biology and Technology, v. 59 (1), p. 53-63, 2011.

ARAGON-ALEGRO, L.C.; ALARCON, J.H. A.; CARDARELLI H. R.; CHIH CHIU, M.; SAAD, S.M. I. Mousse de chocolate potencialmente probiótico e simbiótico. LWT- Food Science and Technology, v.40, p. 669 - 675, 2007.

ARNON-RIPS, H. and POVERENOV, E. Improving food products' quality and storability by using Layer-by-Layer edible coatings. Trends in Food Science and Technology, 2018.

ASBAHANI, A.; MILADI, K.; BADRI, W.; SALA, M.; AIT ADDI, E. H.; CASABIANCA, H.; MOUSADIK, A.; HARTMANN, D.; JILALE, A.; RENAUD, F. N. R.; ELAISSARI, A. Essential oils: From extraction to encapsulation. International Journal of Pharmaceutics, v. 483, p. 220-243, 2015.

ATARÉS, L. and CHIRALT, A. Essential oils as additives in biodegradable films and coatings for active food packaging. Trends in Food Science and Technology, v.48, p. $51-62,2016$.

AUMEERUDDY-ELALFI， Z; GURIB-FAKIM， A.; MAHOMOODALLY, F. Antimicrobial, antibiotic potentiating activity and phytochemical profile of essential oils from exotic and endemic medicinal plants of Mauritius. Industrial Crops and Products, v.71, p. 197-204, 2015.

BALASUNDRAM, N., SUNDRAM, K. and SAMMAN, S. Phenolic compounds in plants and agro-industrial by-products: Antioxidant activity, occurrence, and potential use. Food Chemistry, v.99, p.191-203, 2006.

BALDWIN, E.A.; NISPEROS, M. O.; CHEN, X.; HAGENMAIER, R. D. Improving storage life of cut apple and potato with edible coating. Postharvest Biology and Technology, v.9, p. 151-163, 1996.

BIJI, K. B., RAVISHANKAR, C. N., MOHAN, C. O. and GOPAL, T. K. S. Smart packaging systems for food applications: A review. Journal of Food Science and Technology, v. 52(10), p. 6125-6135, 2015.

BRAGA, F. G.; BOUZADA, M. L. M.; FABRI, R. L.; MATOS, M. D. O.; Antileishmanial and antifungal activity of plants used in traditional medicine in Brazil. Journal off Ethnopharmacology, v. 111 (2), p. 396-402, 2007.

BURT, S. Essential Oils: Their Antibacterial Properties and Potential Applications in Foods. International Journal of Food Microbiology, v. 94, p. 223-253, 2004.

CALLAWAY, T.R.; CARROLL, J. A; ARTHINGTON J.D.; EDRINGTON T. S.; ANDERSON R.C.; RICKE S.C. Citrus products and their use against bacteria: potential health and cost benefits, (chap. 17). R. Watson, J.L. Gerald, V.R. Preedy (Eds.), Nutrients, dietary supplements, and nutriceuticals: Cost analysis versus clinical benefits, Humana Press, New York, NY, p. 277-286, 2011. 
CALO, J. R.; CRANDALL, P. G.; O'BRYAN, C. A.; RICKE, S. C. Essential oils as antimicrobials in food systems - a review. Food Control, v.54, p. 111-119, 2015.

CAVALCANTI, S.; DE SOUZA, M.; CRISTINA, L.; PATROCÍNIO, S.; NALESSO, M.; SIQUEIRA, D. Volatiles composition and extraction kinetics from Schinus terebinthifolius and Schinus molle leaves and fruit. Revista Brasileira de Farmacologia, v.25, p. 356-362, 2015.

DAINELLI, D., GONTARD, N., SPYROPOULOS, D., ZONDERVAN-VAN DEN BEUKEN, E. and TOBBACK, P. Active and intelligent food packaging: Legal aspects and safety concerns. Trends in Food Science and Technology, v.19, 103-112, 2008.

DAMODARAN, S.; PARKIN, K.L.; FENNEMA, O.R. Química de Alimentos. (4 ed) Porto Alegre-Br, Editora Artmed, p. 900, 2010.

DANNENBERG, G. S.; FUNCK, G. D.; MATTEI, F. J.; SILVA, W. P.; FIORENTINI, A. M. Antimicrobial and antioxidant activity of essential oil from pink pepper tree (Schinus terebinthifolius Raddi) in vitro and in cheese experimentally contaminated with Listeria monocytogenes. Innovative Food Science and Emerging Technologies, v.36, p. 120-127, 2016.

DANNENBERG, G. S.; FUNCK, G. D.; CRUXEN, C. E. S.; MARQUES, J. L.; SILVA, W. P.; FIORENTINI, A. M. Essential oil from pink pepper as an antimicrobial component in cellulose acetate film: Potential for application as active packaging for sliced cheese. LWT- Food Science and Technology, v.81, p. 314-318, 2017.

DANNENBERG, G. S., FUNCK G. D; SILVA, W. P.; FIORENTINI, A. M. Essential oil from pink pepper (Schinus terebinthifolius Raddi): Chemical composition, antibacterial activity and mechanism of action. Food Control, v.95, p.115-20, 2019.

DOURADO, M. T. Óleos essenciais e oleoresina da pimenta rosa (Schinus terebinthifolius Raddi): propriedades químicas e biológicas. Tese (Doutorado) - 120f. Programa de Pós-Graduação em Ciência e Tecnologia Agroindustrial. Universidade Federal de Pelotas. Faculdade de Agronomia Eliseu Maciel. Pelotas, 2012.

ESPITIA, P. J. P. "Filmes comestíveis de pectina: propriedades físico-mecânicas e antimicrobianas - revisão". Hydrocolloids alimentares, v. 35: p. 287-296, 2014.

FALGUERA, V., QUINTERO, J. P., JIM_ENEZ, A., MUNOZ, A. and IBARZ, A. Edible films and coatings: structures, active functions and trends in their use. Trends Food Science and Technology, v.22, p. 291-303, 2011.

FDA. Code fed. Regul. (CFR). Title 21 food drugs. Chapter I - food Drug adm. Dep. Heal. Hum. Serv. Subchapter B - food hum. Consum. (Continued), Part 182 - subst. Gen. Recognized as safe (GRAS 2016). Acessado em: 21 out 2018.

GANIARI, S., CHOULITOUDI, E. AND OREOPOULOU, V. Edible and active films and coatings as carriers of natural antioxidants for lipid food. Trends in Food Science and Technology, v.68, p.70-82, 2017.

GARCIA, L.C.; PEREIRA, L.M.; DE LUCA SARANTOPOULOS, C.I.G.; HUBINGER, M.D. Selection of edible starch coating for minimally processed strawberry. Food and Bioprocess Technology, v.3, p. 834-842, 2010. 
GHABRAIE, M.; VU, K. D.; TATA, L.; SALMIERI, S.; LACROIX. M. Antimicrobial effect of essential oils in combinations against five bacteria and their effect on sensorial quality of ground meat. LWT- Food Science and Technology, v.66, p. 332-339, 2016.

GOIS, F.D.; CAIRO, P.L.G.; CANTARELlI, V. DE S.; COSTA, L.C. DO B.; FONTANA, R.; ALLAMAN, I.B. Effect of Brazilian red pepper (Schinus terebinthifolius Raddi) essential oil on performance, diarrhea and gut health of weanling pigs. Livestock Science, v.183, p. 24-27, 2016.

Guidance to The Commission Regulation (UE) No 450/2009 of 29 May 2009 on active and intelligent materials and articles intended to come into contact with food. In Version 10. European commission health and consumers directorate-general directorate esafety of the food chain. E6- innovation and sustainability. Acesado em: 21 out 2018.

HAFSA, J.; SMACH, M. A.; BEN K. S.R.; CHARFEDDINE, B.; LIMEM, K.; MAJDOUB, H. Physical, antioxidant and antimicrobial properties of chitosan films containing Eucalyptus globulus essential oil. LWT - Food Science and Technology, v.68, p. $356-364,2016$.

HAN, Y., YU, M. and WANG, L. Physical and antimicrobial properties of sodium alginate/carboxymethyl cellulose films incorporated with cinnamon essential oil. Food Packaging and Shelf Life, v.15, p. 35 - 42, 2018.

HENRIQUE, C.M.; CEREDA, M. P.; SARMENTO, S. B. S. Características físicas de filmes biodegradáveis produzidos a partir de amidos modificados de mandioca. Ciência e Tecnologia de Alimentos, v. 28, n. 1, p. 231-240, 2008.

HYLDGAARD, M.; MYGIND, T.; MEYER, R. L. Essential oils in food preservation: mode of action, synergies, and interactions with food matrix components. Frontiers in Microbiology, v. 3, 2012.

JUNEJA, V.K.; DWIVEDI H.P.; YAN, X. Novel natural food antimicrobials. Journal of Food Science and Technology, v.3, p. 381-403, 2012.

LI, M.; MUTHAIYAN, A; O'BRYAN, C.A.; GUSTAFSON, J.E.; LI, Y.; CRANDALL, P.G. Use of natural antimicrobials from a food safety perspective for control of Staphylococcus aureus. Current Pharmaceutical Biotechnology, v.12, p. 12401254, 2011.

LÓPEZ-MALO, A.; ALZAMORA, S. M.; GUERRERO S. Natural antimicrobials from plants. S.M. Alzamora, M.S. Tapia, A. López-Malo (Eds.), Minimally processed fruits and vegetables: Fundamental aspects and applications, Aspen Publication, Gaithersburg, MD, p. 237-258, 2000.

MAJID, I.; AHMAD G. N., MOHAMMAD S. D.; NANDA, V. Novel food packaging technologies: Innovations and future prospective. Journal of the Saudi Society of Agricultural Sciences, v. 17, p. 454-462, 2018.

MUTHAIYAN, A.; LIMAYEM, A.; RICKE, S.C. Antimicrobial strategies for limiting bacterial contaminants in fuel bioethanol fermentations. Progress in Energy and Combustion Science, v.37, p. 351-370, 2011.

NANNAPANENI, R.; CHALOVA, V. I.; STORY, R.; WIGGINS K. C.; CRANDALL, P. G. RICKE S. C. Ciprofloxacin-sensitive and ciprofloxacin- 
resistant Campylobacter jejuni are equally susceptible to natural orange oil-based antimicrobials. Journal of Environmental Science and Health, 44 (2009), pp. 571-577

NGUEFACK, J.; TAMGUE, O.; DONGMO, J. L.; DAKOLE, C. D.; LETH, V.; VISMER, H. F.; NKENGFACK, A. E. Synergistic action between fractions of essential oils from Cymbopogon citratus, Ocimum gratissimum and Thymus vulgaris against Penicillium expansum. Food Control, v. 23(2), p. 377- 383, 2012.

OLIVEIRA, R. P.; SCIVITTARO, W. B. Produção de frutos de morango em função de diferentes períodos de vernalização das mudas. Horticultura Brasileira, v. 27, p. 091095, 2008.

PELISSARI, F.M., FERREIRA, D.C., LOUZADA, L.B., DOS SANTOS, F., CORRÊA, A.C., MOREIRA, F.K.V. and MATTOSO, L.H. Starch-based edible films and coatings: An eco-friendly alternative for food packaging. In Starches for Food Application, p. 359-420. Academic Press, 2019.

REALINI, C. E.; MARCOS, B. Active and intelligent packaging systems for a modern society. Meat Science, v. 98(3), p. $404-419,2014$.

RESTUCCIA, D.; SPIZZIRRI, U. G.; PARISI, O. I.; CIRILLO, G.; CURCIO, M.; IEMMA, F. New EU regulation aspects and global market of active and intelligent packaging for food industry applications. Food Control, v.21 (11), p. 1425 - 1435, 2010.

ROJAS-GRAÜ, M. A.; TAPIA, M. S.; MARTÍN-BELLOSO, O. Using polysaccharidebased edible coatings to maintain quality of fresh-cut Fuji apples. LWT-Food Science and Technology, v. 41(1), p. 139-147, 2008.

ROLLER, S.; LUSENGO, J. Developments in natural food preservatives. Agro Food Industry Hi-Tech, v. 8, p. 22-25, 1997.

RUIZ-NAVAJAS, Y., VIUDA-MARTOS, M., SENDRA, E., PEREZ-ALVAREZ, J. A., FERNÁNDEZ-LÓPEZ, J. In vitro antibacterial and antioxidant properties of chitosan edible films incorporated with Thymus moroderi or Thymus piperella essential oils. Food Control, v. 30, p. 386-392, 2013.

SÁNCHEZ-GONZÁLEZ， L.; PASTOR， C.; VARGAS， M.; CHIRALT, A.; GONZÁLEZ-MARTÍNEZ, C.; CHÁFER, M. Effect of hydroxypropylmethylcellulose and chitosan coating with and without bergamot essential oil on quality and safety of cold-stored grapes. Postharvest Biology and Technology, v. 60, n.1, p. 57-63, 2011.

SIRSAT, S. A.; MUTHAIYAN A.; RICKE S. C. Antimicrobials for foodborne pathogen reduction in organic and natural poultry production. Journal of Applied Poultry Research, v.18, p. 379-388, 2009.

SUNG S.Y.; SIN, L.T.; TEE, T. T.; BEE, S. T.; RAHMAT, A.R.; RAHMAN, W. Antimicrobial agents for food packaging applications. Trends in Food Science and Technology, v.33 (2), p. 110-123, 2013.

SUKHIJA, S.; SINGH, S.; RIAR, C. S. Isolation of starches from different tubers and study of their physicochemical, thermal, rheological and morphological characteristics. Starch-Stärke, v.68 (1-2), p.160-168, 2016. 
TI, F. M.; FERREIRA, D. C., LOUZADA, L. B., DOS SANTOS, F., CORRÊA, A. C., MOREIRA, F. K. V. and MATTOSO, L. H. Starch-Based Edible Films and Coatings: An Eco-friendly Alternative for Food Packaging. Starches for Food Application. Academic Press, pp. 359-420, 2019.

TIWARI, B. K.; VALDRAMIDIS, V. P.; O'DONNELL C. P.; MUTHUKUMARAPPAN, K.; BOURKE, P.; CULLEN. P. J. Application of natural antimicrobials for food preservation. Journal of Agricultural and Food Chemistry, v.57, p. 5987-6000, 2009.

TZOUMAKI, M. V.; BILIADERIS, C. G.; VASILAKAKIS, M. Impact of edible coatings and packaging on quality of white asparagus (Asparagus officinalis, L.) during cold storage. Food Chemistry, v.117, p. 55 - 63, 2009.

ULIANA, M. P.; FRONZA, M.; DA SILVA, A. G.; VARGAS, T. S.; DE ANDRADE, T. U.; SCHERER, R. Composition and biological activity of Brazilian rose pepper (Schinus terebinthifolius Raddi) leaves. Industrial Crops and Products, v.83, p. 235240, 2016.

VILELA, C.; KUREK, M.; HAYOUKA, Z.; ROCKER, B.; YILDIRIM, S.; ANTUNES, M. D. C.; NILSEN-NYGAARD, J.; PETTERSEN, M. K.; FREIRE, C. S. R. A concise guide to active agents for active food packaging - Review. Trends in Food Science and Technology. v. 80, p. 212-222, 2018.

VIUDA-MARTOS, M.; EL GENDY, N. G. S.; SENDRA, E.; FERNANDEZ-LOPEZ, J.; EL- RAZIK, K. A. A.; EL-SAYED, A. Chemical composition and antioxidant and antilisteria activities of essential oils obtained from some Egyptian plants. Journal of Agricultural and Food Chemistry, v.58, p. 9063-9070, 2010.

VU, K. D.; HOLLINGSWORTH R. G.; LEROUX, E.; SALMIERI, S.; LACROIX, M. Development of edible bioactive coating based on modified chitosan for increasing the shelf life of strawberries. Food Research International, v.44, p. 198-203, 2011.

WONG, W. S.; TILLIN, S. J.; HUDSON, J.S.; PAVLATH, A. E. Gas exchange in cut apples with bilayer coatings. Journal of Agricultural and Food Chemistry, v.42, p. 2278-2285, 1994.

WRÓBLEWSKA-KREPSZTUL， J.; RYDZKOWSKI， T.; BOROWSKI， G.; SZCZYPIŃSKI, M.; KLEPKA, T.; THAKUR, V. K. Recent progress in biodegradable polymers and nanocomposite-based packaging materials for sustainable environment. International Journal of Polymer Analysis and Characterization, v. 23(4), p. 383-395, 2018.

YILDIRIM, S.; RÖCKER, B.; PETTERSEN, M. K.; NILSEN-NYGAARD, J.; AYHAN, Z.; RUTKAITE, R. Active packaging applications for food. Comprehensive Reviews. Food Science and Food Safety, v.17, p.165-199, 2018.

YANG, H. J.; LEE, J. H.; WON, M.; SONG, K. B. Antioxidant activities of distiller-dried grains with solubles as protein films containing tea extracts and their application in the packaging of pork meat. Food Chemistry, v.196, p. 174 -179, 2016.

ZAHID, N. Z.; ABBASI, N. A.; HAFIZ, A. I.; HUSSAIN, A.; AHMAD, Z. Antifungal activity of local fennel (Foeniculum vulgare Mill) extracts to growth responses of some soil diseases. Afr. J. Microbiol. Res, v.6, p. 46-51, 2012. 
ZANETTI, M.; TERNUS, Z. R.; DALCANTON, F.; DE MELLO, M. M. J.; DE OLIVEIRA, D.; ARAUJO, P. H. Microbiological characterization of pure geraniol and comparison with bactericidal activity of the cinnamic acid in gram-positive and gramnegative bacteria. Journal of Microbial and Biochemical Technology, v.7, p.186-193, 2015. 